\title{
In Metric-measure Spaces Sobolev Embedding is Equivalent to a Lower Bound for the Measure
}

\author{
Przemysław Górka1
}

Received: 10 April 2015 / Accepted: 14 November 2016 / Published online: 22 November 2016

(C) The Author(s) 2016. This article is published with open access at Springerlink.com

\begin{abstract}
We study Sobolev inequalities on doubling metric measure spaces. We investigate the relation between Sobolev embeddings and lower bound for measure. In particular, we prove that if the Sobolev inequality holds, then the measure $\mu$ satisfies the lower bound, i.e. there exists $b$ such that $\mu(B(x, r)) \geq b r^{\alpha}$ for $r \in(0,1]$ and any point $x$ from metric space.
\end{abstract}

Keywords Sobolev spaces - Sobolev inequalities - Metric measure spaces · Lower bound for measure

Mathematics Subject Classifications (2010) 46E35 · 30L99

\section{Introduction}

Let $\Omega$ be an open subset of the Euclidean space $\mathbb{R}^{n}$. If the boundary of $\Omega$ is sufficiently regular and $1 \leq p<n$, then the Sobolev embedding $W^{1, p}(\Omega) \hookrightarrow L^{p^{*}}(\Omega)$ holds, where $p^{*}:=\frac{n p}{n-p}$ (see e.g. [1]). On the other hand, it was shown by Hajłasz-Koskela-Tuominen [7] that if $W^{1, p}(\Omega) \hookrightarrow L^{p^{*}}(\Omega)$, then $\Omega$ satisifies the so-called measure density condition, i.e. there exists a constant $c>$ such that for all $x \in \Omega$ and all $0<r \leq 1$

$$
|B(x, r) \cap \Omega| \geq c r^{n} .
$$

More recently, the result of Hajłasz-Koskela-Tuominen has been extended to the Slobodeckij-Sobolev spaces $W^{s, p}$ (see Zhou [12]). Namely, if $W^{s, p}(\Omega) \hookrightarrow L^{n p /(n-s p)}(\Omega)$ for some $s \in(0,1)$ and $p \geq 1$ such that $s p<n$, then $\Omega$ satisfies (1).

Przemysław Górka

pgorka@mini.pw.edu.pl

1 Department of Mathematics, Information Sciences, Warsaw University of Technology, Ul.

Koszykowa 75, Warsaw, 00-662, Poland 
The main objective of the paper is to study the relation between Sobolev inequalities on metric measure spaces and lower bound for measure. In particular, we prove the following result. Suppose that $(X, \rho, \mu)$ is a metric measure space equipped with doubling measure and let $M^{1, p}(X)$ be the Hajłasz-Sobolev space. If $M^{1, p}(X) \hookrightarrow L^{q}(X)$, where $p<q$, then there exists $b>0$ such that for any $x \in X$ and $0<r \leq 1$, the following inequality holds

$$
\mu(B(x, r)) \geq b r^{\alpha},
$$

where $\frac{1}{p}-\frac{1}{q}=\frac{1}{\alpha}$.

The remainder of the paper is structured as follows. In Section 2, we introduce the notations and recall the notion of Sobolev spaces on general metric measure spaces. Our principal assertion, concerning the sufficient and necessary conditions for Sobolev embeddings are formulated and proven in Section 3.

\section{Preliminaries}

Let $(X, \rho, \mu)$ be a metric measure space equipped with a metric $\rho$ and the Borel regular measure $\mu$. We assume throughout the paper that the measure of every open nonempty set is positive and that the measure of every bounded set is finite. Additionally, we assume that the measure $\mu$ satisfies a doubling condition. It means that, there exists a constant $C_{d}>0$ such that for every ball $B(x, r)$,

$$
\mu(B(x, 2 r)) \leq C_{d} \mu(B(x, r)) .
$$

It is well known (see e.g. Lemma 8.1.13 in [9]) that the doubling condition implies that, there exists a positive constant $D$ satisfying

$$
D\left(\frac{r_{1}}{r_{2}}\right)^{s} \leq \frac{\mu\left(B\left(x_{1}, r_{1}\right)\right)}{\mu\left(B\left(x_{2}, r_{2}\right)\right)}, \quad \text { where } s=\log _{2} C_{d},
$$

for all balls $B\left(x_{2}, r_{2}\right)$ and $B\left(x_{1}, r_{1}\right)$ with $r_{2} \geq r_{1}>0$ and $x_{1} \in B\left(x_{2}, r_{2}\right)$. It follows from the above inequality that if $X$ is bounded, then there exists $b>0$ such that the following inequality holds for $r<\operatorname{diam} X$

$$
\mu(B(x, r)) \geq b r^{s} .
$$

On the other hand, if the metric measure space equipped with a doubling measure is not bounded, then inequality (2) does not necessarily hold.

Furthermore, we need to recall the notion of Ahlfors regularity. We shall say that the metric measure space $(X, \rho, \mu)$ is Ahlfors $s$-regular if there exist constants $b$ and $B$ such that

$$
b r^{s} \leq \mu(B(x, r)) \leq B r^{s}
$$

for all balls $B(x, r) \subset X$ with $r<\operatorname{diam} X$.

We are now in a position to recall the notion of Sobolev spaces on metric measure spaces (see also [4]). Let $(X, \rho, \mu)$ be a metric measure space. We say that a $p$-integrable function $f$ belongs to the Hajłasz-Sobolev space $M^{1, p}(X)$ if there exists non-negative $g \in L^{p}(X)$, called a generalized gradient, such that

$$
|f(x)-f(y)| \leq \rho(x, y)(g(x)+g(y)) \quad \text { a.e. for } \quad x, y \in X .
$$

We equip the space $M^{1, p}(X)$ with the norm

$$
\|f\|_{M^{1, p}(X)}=\|f\|_{L^{p}(X)}+\inf \|g\|_{L^{p}(X)},
$$


where the infimum is taken over all the generalized gradients. Then $M^{1, p}$ is a Banach space. For the basic properties of this kind of spaces, we refer to [2, 4-6, 9-11].

Suppose that $f$ is locally integrable and $A$ is a measurable set of positive measure, then by $f_{A}$ we denote the integral average of the function $f$ over the set $A$, i.e.,

$$
f_{A}:=f_{A} f d \mu=\frac{1}{\mu(A)} \int_{A} f d \mu .
$$

\section{Main Results}

In this section, we show the sufficient and necessary conditions for Sobolev inequalities. We will start with the following proposition.

Proposition 3.1 Let $(X, \rho, \mu)$ be a metric measure space with s-regular measure $\mu$. If $s>p \geq 1$, then

$$
M^{1, p}(X) \hookrightarrow L^{p^{*}}(X)
$$

where $p^{*}=\frac{s p}{s-p}$. Moreover, there exists $C=C(s, p, b)$, depending on $s, p, b$, such that for each $u \in M^{1, p}(X)$, the following inequality holds

$$
\|u\|_{L^{p^{*}}(X)} \leq C\left(\|u\|_{L^{p}(X)}+\|g\|_{L^{p}(X)}\right) .
$$

Furthermore, if $\operatorname{diam} X=\infty$, then

$$
\|u\|_{L^{p^{*}}(X)} \leq C\|g\|_{L^{p}(X)} .
$$

Proof Taking $\sigma=2$ in Theorem 8.7 from [5], we have

$$
\left(f_{B\left(x_{0}, r\right)}\left|u-u_{B\left(x_{0}, r\right)}\right|^{p^{*}} d \mu\right)^{\frac{1}{p^{*}}} \leq C\left(\frac{\mu\left(B\left(x_{0}, 2 r\right)\right)}{b r^{s}}\right)^{\frac{1}{p}} r\left(f_{B\left(x_{0}, 2 r\right)} g^{p} d \mu\right)^{\frac{1}{p}},
$$

where the constant $C$ depends on $p$ and $s$. Hence, we get

$$
\begin{aligned}
\left(\int_{B\left(x_{0}, r\right)}|u|^{p^{*}} d \mu\right)^{\frac{1}{p^{*}}} & \leq\left(\int_{B\left(x_{0}, r\right)} \mid u_{\left.\left.B\left(x_{0}, r\right)\right|^{p^{*}} d \mu\right)^{\frac{1}{p^{*}}}}\right. \\
& +C b^{-\frac{1}{p}} \frac{\mu\left(B\left(x_{0}, r\right)\right)^{\frac{1}{p^{*}}}}{r^{\frac{s}{p^{*}}}}\left(\int_{B\left(x_{0}, 2 r\right)} g^{p} d \mu\right)^{\frac{1}{p}} .
\end{aligned}
$$

Thus, by the Hölder inequality, we obtain

$$
\begin{array}{r}
\left(\int_{B\left(x_{0}, r\right)}|u|^{p^{*}} d \mu\right)^{\frac{1}{p^{*}}} \leq \frac{b^{-\frac{1}{s}}}{r}\left(\int_{B\left(x_{0}, r\right)}|u|^{p} d \mu\right)^{\frac{1}{p}}+C b^{-\frac{1}{p}} B^{\frac{1}{p^{*}}}\left(\int_{B\left(x_{0}, 2 r\right)} g^{p} d \mu\right)^{\frac{1}{p}} \leq \\
\frac{b^{-\frac{1}{s}}}{r}\left(\int_{X}|u|^{p} d \mu\right)^{\frac{1}{p}}+C b^{-\frac{1}{p}} B^{\frac{1}{p^{*}}}\left(\int_{X} g^{p} d \mu\right)^{\frac{1}{p}} .
\end{array}
$$

Finally, by passing to $\operatorname{diam} X$ with $r$, we obtain the desired result.

Next, we state necessary conditions for Sobolev embeddings. The proof of the following theorem relies on the methods established by Carron (see [3] and proof of Lemma 2.2 in [8]). 
Theorem 3.2 Suppose that $(X, \rho, \mu)$ is a metric measure space with the doubling measure. If

$$
M^{1, p}(X) \hookrightarrow L^{q}(X),
$$

where $q>p$, then there exists $b=b\left(p, q, C_{p q}\right)$ such that

$$
\mu(B(x, r)) \geq b r^{\alpha}, \quad \text { for } \quad r \in(0,1],
$$

where $\frac{1}{p}-\frac{1}{q}=\frac{1}{\alpha}$ and $C_{p, q}$ is the constant of the embedding.

Remark 1 Let us stress that $b$ does not depend on the doubling constant $C_{d}$.

Proof For each $u \in M^{1, p}(X)$ we have

$$
\left(\int_{X}|u|^{q} d \mu\right)^{\frac{1}{q}} \leq C_{p q}\left(\left(\int_{X}|u|^{p} d \mu\right)^{\frac{1}{p}}+\left(\int_{X} g^{p} d \mu\right)^{\frac{1}{p}}\right),
$$

where $g$ is a generalized gradient of $u$. For a fixed $x \in X$ and $R>0$, let us define a Lipschitz function $u_{R}$ as follows

$$
u_{R}(y):= \begin{cases}\frac{2}{R}(R-\rho(y, x)) & \text { if } y \in B(x, R) \backslash B\left(x, \frac{R}{2}\right) \\ 1 & \text { if } y \in B\left(x, \frac{R}{2}\right) \\ 0 & \text { if } y \in X \backslash B(x, R),\end{cases}
$$

It is easily seen that as a generalized gradient we can take

$$
g_{R}(y)=\frac{2}{R} \chi_{B(x, R)}
$$

Since the support of $u$ is contained in $B(x, R)$, we get in view of the Hölder inequality

$$
\left(\int_{X}\left|u_{R}\right|^{p} d \mu\right)^{\frac{1}{p}} \leq \mu(B(x, R))^{\frac{1}{\alpha}}\left(\int_{X}\left|u_{R}\right|^{q} d \mu\right)^{\frac{1}{q}} .
$$

Therefore, we have

$$
\frac{1}{\mu(B(x, R))^{\frac{1}{\alpha}}}-C_{p q} \leq C_{p q} \frac{\left(\int_{X} g_{R}^{p} d \mu\right)^{\frac{1}{p}}}{\left(\int_{X}\left|u_{R}\right|^{p} d \mu\right)^{\frac{1}{p}}} .
$$

Let us fix $r \leq 1$ and $x \in X$. Then, $\mu(B(x, r)) \geq\left(\frac{1}{2 C_{p q}}\right)^{\alpha} r^{\alpha}$ or $\mu(B(x, r)) \leq\left(\frac{1}{2 C_{p q}}\right)^{\alpha} r^{\alpha}$. In the first case we have the desired inequality. Thus we may assume that $\mu(B(x, r)) \leq$ $\left(\frac{1}{2 C_{p q}}\right)^{\alpha} r^{\alpha}$. In this case, we get for any $\delta \leq r$

$$
\mu(B(x, \delta)) \leq\left(\frac{1}{2 C_{p q}}\right)^{\alpha} .
$$

Due to (3), we have

$$
\frac{1}{\left(2 C_{p q}\right)^{p}} \mu(B(x, \delta))^{-\frac{p}{\alpha}} \leq \frac{\int_{X} g_{\delta}^{p} d \mu}{\int_{X}\left|u_{\delta}\right|^{p} d \mu} .
$$

Consequently, the structure of $u_{\delta}$ and $g_{\delta}$ implies the following inequality

$$
\frac{1}{\left(2 C_{p q}\right)^{p}} \mu(B(x, \delta))^{-\frac{p}{\alpha}} \leq \frac{\left(\frac{2}{\delta}\right)^{p} \mu(B(x, \delta))}{\mu\left(B\left(x, \frac{\delta}{2}\right)\right)} .
$$


Hence, we obtain the following estimate

$$
\mu(B(x, \delta)) \geq\left(\frac{\delta}{4 C_{p q}}\right)^{\frac{\alpha p}{p+\alpha}}\left(\mu\left(B\left(x, \frac{\delta}{2}\right)\right)\right)^{\frac{\alpha}{p+\alpha}}
$$

for each $\delta \leq r$. Thus, Theorem 3.2 easily follows from the above estimate and the doubling condition, but we wish to obtain the constant $b$ independent of the doubling constant $C_{d}$, so more work is required.

In order to reach our goal, we iterate inequality (4) and we get for any integer $n$

$$
\mu(B(x, r)) \geq\left(\frac{r}{2 C_{p q}}\right)^{p \sum_{j=1}^{n}\left(\frac{\alpha}{p+\alpha}\right)^{j}}\left(\frac{1}{2}\right)^{p \sum_{j=1}^{n} j\left(\frac{\alpha}{p+\alpha}\right)^{j}}\left(\mu\left(B\left(x, \frac{r}{2^{n}}\right)\right)\right)^{\left(\frac{\alpha}{p+\alpha}\right)^{n}} .
$$

On the other hand, since the measure is doubling, we have

$$
(\mu(B(x, r)))^{\left(\frac{\alpha}{p+\alpha}\right)^{n}} \geq\left(\mu\left(B\left(x, \frac{r}{2^{n}}\right)\right)\right)^{\left(\frac{\alpha}{p+\alpha}\right)^{n}} \geq C_{d}^{-n\left(\frac{\alpha}{p+\alpha}\right)^{n}}(\mu(B(x, r)))^{\left(\frac{\alpha}{p+\alpha}\right)^{n}},
$$

hence

$$
\lim _{n \rightarrow \infty}\left(\mu\left(B\left(x, \frac{r}{2^{n}}\right)\right)\right)^{\left(\frac{\alpha}{p+\alpha}\right)^{n}}=1
$$

Thus, letting $n \rightarrow \infty$ in (5), we obtain

$$
\mu(B(x, r)) \geq \frac{1}{\left(2 C_{p q}\right)^{\alpha} 2^{\frac{\alpha(\alpha+p)}{p}}} r^{\alpha} .
$$

This completes the proof of our claim.

Now, as a corollary from the proof of Theorem 3.2 and Proposition 3.1, we obtain the following theorem.

Theorem 3.3 Suppose that $(X, \rho, \mu)$ is an unbounded metric measure space with the doubling measure such that $\mu(B(x, r)) \leq B r^{\alpha}$ for all $r>0$ and $x \in X$. Assume that $1 \leq p<q$ and $\alpha=\frac{p q}{q-p}$. Then, the following conditions are equivalent:

i) there exists $b>0$ such that for any $x \in X$ and $r>0$, the following inequality holds

$$
\mu(B(x, r)) \geq b r^{\alpha},
$$

ii)

$$
M^{1, p}(X) \hookrightarrow L^{q}(X),
$$

and, there exists $C>0$ such that for each $u \in M^{1, p}(X)$ the following inequality holds

$$
\|u\|_{L^{q}(X)} \leq C\|g\|_{L^{p}(X)} .
$$

Proof Implication $i$ ) to $i i$ ) follows directly from Proposition 3.1. Now, we shall prove the converse. Let us assume that $i i$ ) holds. In other words, for any $u \in M^{1, p}(X)$ we have

$$
\left(\int_{X}|u|^{q} d \mu\right)^{\frac{1}{q}} \leq C\left(\int_{X} g^{p} d \mu\right)^{\frac{1}{p}},
$$


where $g$ is a generalized gradient of $u$. Next, we use the techniques from the proof of Theorem 3.2. We have

$$
\frac{1}{\mu(B(x, r))^{\frac{1}{\alpha}}} \leq C \frac{\left(\int_{X} g_{r}^{p} d \mu\right)^{\frac{1}{p}}}{\left(\int_{X}\left|u_{r}\right|^{p} d \mu\right)^{\frac{1}{p}}} .
$$

Hence, we obtain the following estimate

$$
\mu(B(x, r)) \geq\left(\frac{r}{2 C}\right)^{\frac{\alpha p}{p+\alpha}}\left(\mu\left(B\left(x, \frac{r}{2}\right)\right)\right)^{\frac{\alpha}{p+\alpha}} .
$$

Iteration of the above inequality leads us to the estimate

$$
\mu(B(x, r)) \geq\left(\frac{r}{C}\right)^{p \sum_{j=1}^{n}\left(\frac{\alpha}{p+\alpha}\right)^{j}}\left(\frac{1}{2}\right)^{p \sum_{j=1}^{n} j\left(\frac{\alpha}{p+\alpha}\right)^{j}}\left(\mu\left(B\left(x, \frac{r}{2^{n}}\right)\right)\right)^{\left(\frac{\alpha}{p+\alpha}\right)^{n}} .
$$

Finally, letting $n \rightarrow \infty$ in the previous inequality, we get

$$
\mu(B(x, r)) \geq \frac{1}{(C)^{\alpha} 2^{\frac{\alpha(\alpha+p)}{p}}} r^{\alpha},
$$

which completes the proof of Theorem 3.3.

Furthermore, we have the following equivalence.

Theorem 3.4 Suppose that $(X, \rho, \mu)$ is a bounded metric measure space with the doubling measure. Assume that $1 \leq p<q$ and $\alpha=\frac{p q}{q-p}$. Then, the following conditions are equivalent:

i) there exists $b>0$ such that for any $x \in X$ and $0<r<\operatorname{diam} X$, the following inequality holds

$$
\mu(B(x, r)) \geq b r^{\alpha},
$$

ii)

$$
M^{1, p}(X) \hookrightarrow L^{q}(X)
$$

Proof Implication from $i$ ) to $i$ i) follows from Theorem 6 in [4]. Next, let us assume that $M^{1, p}(X) \hookrightarrow L^{q}(X)$, then from Theorem 3.2 we have

$$
\mu(B(x, r)) \geq b r^{\alpha}, \quad \text { for } \quad r \in(0,1] .
$$

From the above estimate, we get for $0<r<\operatorname{diam} X$ the following inequality

$$
\mu(B(x, r)) \geq \mu\left(B\left(x, \frac{r}{\operatorname{diam} X+1}\right)\right) \geq b\left(\frac{r}{\operatorname{diam} X+1}\right)^{\alpha}=C r^{\alpha},
$$

and the proof is complete.

Open problem 1 The doubling condition plays a role in the proofs of Theorem 3.2 and Theorem 3.3, but not in the statements of the results. Hence, it is natural to state the following problem. Are the results true without the doubling condition? 
Acknowledgments Some part of the paper has been performed during the visit of the author to the TIFR Centre for Applicable Mathematics in Bangalore. P.G. wish to thank for its hospitality. The research was partially supported by a grant Iuventus Plus of the Ministry of Science and Higher Education of the Republic of Poland, Nr 0009/IP3/2015/73. The author would like to thank the referee for detailed reports and many constructive suggestions and comments. Moreover, the author thanks Marcin Dudziński for reading the preliminary version of this manuscript.

Open Access This article is distributed under the terms of the Creative Commons Attribution 4.0 International License (http://creativecommons.org/licenses/by/4.0/), which permits unrestricted use, distribution, and reproduction in any medium, provided you give appropriate credit to the original author(s) and the source, provide a link to the Creative Commons license, and indicate if changes were made.

\section{References}

1. Adams, R.A.: Sobolev Spaces, vol. 65. Academic Press, New York-London (1975)

2. Ambrosio, L., Tilli, P.: Topics on Analysis in Metric Spaces, vol. 25. Oxford University Press, Oxford (2004)

3. Carron, G.: Inégalités isopérimétriques et inégalités de Faber-Krahn, Séminaire de Théorie Spectrale et Géométrie, No. 13, Année 1994-1995, 63-66

4. Hajłasz, P.: Sobolev spaces on an arbitrary metric space. Potential Anal. 5, 403-415 (1996)

5. Hajłasz, P.: Sobolev spaces on metric-measure spaces, Heat kernels and analysis on manifolds, graphs, and metric spaces (Paris, 2002). Contemp. Math. 338, 173-218 (2003)

6. Hajłasz, P., Koskela, P.: Sobolev met Poincaré. Memoirs Amer. Math. Soc. 688, 1-101 (2000)

7. Hajłasz, P., Koskela, P., Tuominen, H.: Sobolev embeddings, extensions and measure density condition. J. Funct. Anal. 254, 1217-1234 (2008)

8. Hebey, E.: Sobolev spaces on riemannian manifolds. springer. lecture notes in mathematics 1635 (1996)

9. Heinonen, J., Juha, Koskela, P., Shanmugalingam, N., Tyson, J.T.: Sobolev spaces on metric measure spaces. Cambridge University Press, Cambridge (2015)

10. Heinonen, J.: Lectures on analysis on metric spaces, universitext (2001)

11. Kinnunen, J., Martio, O.: The Sobolev capacity on metric spaces. Ann. Acad. Sci. Fenn. Math. 21, 367-382 (1996)

12. Zhou, Y., Fractional Sobolev extension and imbedding: Trans. Amer. Math Soc. 367, 959-979 (2015) 